\title{
Rabies encephalitis in children: a resurgence of a fatal anthropozoonosis
}

\author{
Fatma Khalsi, Ahmed Ayari, Manel Ben Romdhane, Faten Tinsa, Khedija Boussetta
}

Service de Médecine Infantile B, Hôpital d'Enfants Béchir Hamza de Tunis, Tunisie.

DOI: https://dx.doi.org/10.4314/ahs.v18i3.10

Cite as: Khalsi F, Ayari A, Romdhane MB, Tinsa F, Boussetta K. Rabies encephalitis in children: a resurgence of a fatal anthropozoonosis. Afri Health Sci. 2018;18(3): 539-541. bttps:/ / dx.doi.org/10.4314/abs.v18i3.10

\section{Introduction}

Rabies is a ubiquitous fatal disease and its large wild life reservoir may precipitate a flare-up whenever stringent control is relaxed. It remains a serious public health challenge in the developing world. It is estimated that up to 40-60,000 cases of human rabies occur annually, mostly in rural areas of Africa and Asia, and with a particularly high incidence in young children (under the age of 15 years). Although Tunisia has implemented a national program against rabies since 1982, we are still recording one or two cases every year; in these cases, the victims generally did not consult after a dog bite and did not receive prophylaxis.

We report in this article the case of an eleven year old child, who received post exposure prophylaxis and unfortunately developed fatal rabies.

\section{Case report}

An eleven year old boy was brought to the emergency department for fever, vomiting and acute speech troubles, he had no remarkable medical history and his developmental milestones were normal. On physical examina-

\section{Corresponding author:}

Fatma Khalsi,

Service de Médecine Infantile B, Hôpital d'Enfants Béchir Hamza de Tunis, Tunisie.

Tel :0021698255935

E-mail: khalsif@gmail.com tion, he was febrile at $38.5^{\circ} \mathrm{c}$, he had no signs of meningeal irritation; he had strabismus, dysarthria, tremor, deep tendon reflexes were very weak. Noteworthy, he had an intense thirst and desire to drink, but he was unable to swallow because of pharyngeal spasms.

Cerebral CT scan was performed and did not show any abnormalities. Blood count revealed neutrophilic leucocytosis; normal $\mathrm{C}$ reactive protein and normal electrolytes levels were found.

On further questioning, his mother revealed a history of a stray dog bite two weeks prior. The patient was bitten in his face (lips and the inner cheek); wounds were sutured and he received 12 hours later, a maximum dose of equine rabies immunoglobulin (ERIG) 40UI/kg (Pasteur) into the wound and the rest intramuscularly into the deltoid region on day 0 after wound cleaning and four doses of rabies vaccine ( purified chicken embryo rabies vaccine PCEC, Rabipur, Novartis vaccines) intramuscularly into the deltoid region on days: 0, 3, 7 and 14 following WHO guidelines (ESSEN regimen+ERIG).

Four hours after admission, he became furious, and developed hyper-salivation. His condition deteriorated and he succumbed to death although he was intubated and ventilated. Post-mortem cerebrospinal fluid showed a pleocytosis with 400 leucocytes (80\% lymphocytes).

On autopsy, biopsies of cerebral cortex, cerebellum, hippocampus and medulla were positive for rabies antigen on fluorescent antibody technique.

\section{Discussion}

Rabies is fatal encephalitis of viral origin in humans and 
some other mammals. It is estimated that up to 40-60,000 cases of human rabies occur annually, and a dog bite is the cause of over $98 \% .^{1}$ It is still a major health problem in the developing world and has medical and economic implications: nearly 4 million people receive post-exposure prophylaxis (PEP) annually.

In Tunisia, rabies PEP (RPEP; vaccine+immunoglobulin) is free of charge and is included in the national rabies control programme. There are specialised centres in many regions of our country for RPEP.

One or two cases of fatal human rabies have been recognised per year in Tunisia; in these cases, the victims generally did not consult after a dog bite and did not receive prophylaxis.

True prophylaxis failures are rare and the most cases of RPEP management failure are due to errors of management.

Like the Lyssa virus, rabies virus is a neurotropic virus, clinical manifestations begin at least five to six days after the virus has reached the central nervous system ${ }^{4}$.

We recognize two forms of human rabies, the well known classical form or encephalitic (furious) that occurs in 70\% of the cases and the paralytic (dumb) rabies in 30\% of clinical presentations.

Clinical features of the disease can be devided into five stages: incubation period,prodrome phase, the acute neurologic phase and coma then death ${ }^{5}$. Incubation phase usually lasts between 20 to 60 days, shorter and longer periods has been reported (from few days to 19 years). The post exposure prophylaxis is effective only during the incubation phase and should be administered as soon as possible. The prodromic phase is characterized by non specific symptoms that include fever, malaise, pharyngitis and paraesthesias at the site of the bite.

During the encephalitic phase patients may present with fluctuating consciousness with intermittent episodes of confusion, hallucinations, agitation or aggressive behavior ${ }^{6}$. Signs of autonomic dysfunction are also observed, with hyper-salivation, dilated pupils, piloerection or priapism. Hydrophobia (in $50 \%$ to $80 \%$ of furious form) is a characteristic manifestation of rabies and may be associated with aerophobia which can be elicited by the "fan test". This is followed by paralysis and coma. Death is usually due to respiratory failure, which occurs at a mean of five days after onset of symptoms, in the absence of intensive medical care.
Paralytic form closely mimics Guillain Barré syndrome and may not be recognized by physicians. Death is caused by respiratory failure and occurs in about 2 weeks.

Once rabies encephalitis develops, no therapy has proved effective. Recently some cases of survivals have been reported $^{7,8,9,10}$; but it is still unknown whether recovery resulted from therapy administrated to the patients or whether survival resulted from effective endogenous immune response in combination with the massive critical care support implemented during hospitalization ${ }^{10}$.

Given the fatal outcome of the disease, prevention should be implemented in endemic zones. It consists of vaccinating animals, providing post-exposure prophylaxis and especially spreading awareness among the targeted population.

In our case, although post exposure prophylaxis was established in an optimal way, the wound was sutured which represents a second inoculation of rabies virus and increased the chances to develop lethal encephalitis.

Post-exposure prophylaxis (PEP) is not always effective. Treatment failure reported in the literature ${ }^{8-11}$ involved wounds in highly innerved regions in the body such as face wounds which is the case in this child (wound in lip and cheek), or errors in the management like: inappropriate wound cleaning, or the fact that the wound has been sutured before immunoglobulin injection, or may be some of the wounds have been missed and not injected with immunoglobulin or immunoglobulin injection was administered too late (12 hours after the bite in this case). This case alerts the physician about the importance of the first aid of the wound and of not stitching the wound before immunoglobulin injection. The extensive wound washing which is not well known by physicians (immediate and thorough flushing and washing of the wound for a minimum of 15 minutes with soap and water, detergent, povidone iodine or other substances that kill the rabies virus...) could have avoided the failure of immunoglobulin and vaccine and prevented rabies in this child ${ }^{6}$.

Physicians must be aware of the clinical characteristics of rabies and know how to prescribe vaccines and ERIG appropriately. Rabies encephalitis is a fatal disease and animal rabies control and surveillance are mandatory, especially in endemic areas. Our case has many learning points (clinical characteristic of rabies and rabies post exposure prophylaxis). It shows variability and non-specif- 
ic signs of rabies encephalitis which are frequently not recognized by physicians (fever, non specific neurological signs, normal CT scan, CSF pleiocyosis), it also alerts the physicians in endemic areas to evoke human rabies when the patient presents with encephalitis signs even when he/she has received "adequate" PEP after a dog bite.

\section{Conflict of interest}

None.

\section{Acknowledgments}

F.Khalsi and A.Ayari: wrote the manuscript. F.Tinsa: contributed to the management of the patient. K.Boussetta: corrected the manuscript.

\section{References}

1. International Committee on Taxonomy of Viruses (ICTV). Virology Division-IUMS. http://www.ictvonline.org/index.asp? bhcp=1. (Accessed October 4, 2010). 2. Dacheux L., Delmasand O., Bourhy H. Human Rabies Encephalitis Prevention and Treatment: Progress Since Pasteur's Discovery. Infectious Disorders - Drug Targets 2011;11:251-299.

3. Delmas, O., Holmes E.C., Talbi C.,Larrous F.,Dacheux L., Bouchier C. et al. Genomic diversity and evolution of the lyssaviruses. PLoS One. 2008; 3(4): e2057. PubMed
4. Hemachudha T., Laothamatas J., Rupprecht C.E. Human rabies: a disease of complex neuropathogenetic mechanisms and diagnostic challenges. Lancet Neurol. 2002; 1(2):101-109. PubMed.

5. Dupont JR. and Kenneth M.Human rabies encephalitis A study of forty-nine fatal cases with a review of the literature. Earle Neurology. 1965;15:1023.

6. World Health Organization. WHO Expert Consultation on Rabies. Second report. World Health Organ Tech Rep Ser. 2013;982:1-139.

7. Mani RS, Madhusudana SN, Mahadevan A, et al. Utility of real-time Taqman PCR for antemortem and postmortem diagnosis of human rabies. J Med Virol. 2014;86:1804-12. PubMed.

8. Wilde H. Failures of post-exposure rabies prophylaxis. Vacine. 2007;25:7605-9. PubMed.

9. Hemachudha T, Mitrabhakdi E, Wilde H, et al.Additional reports of failure to respond to treatment after rabies exposure in Thailand. Clin Infect Dis. 1999;28:143-4. 10. Shantavasinkul P, Tantawichien T, Wacharapluesadee $\mathrm{S}$, et al. Failure of rabies post exposure prophylaxis in patients presenting with unusual manifestations. Clin Infect Dis. 2010;50:77- 9. PubMed.

11.Tinsa F, Borgi A, Jahouat I, Boussetta K.Rabies encephalitis in a child: a failure of rabies post exposure prophylaxis. BMJ Case Rep. Jan 14;2015. pii: bcr2014206191. doi: 10.1136/bcr-2014-20619. 